\title{
Cuantificación del desempeño económico de los gobiernos locales en México
}

\author{
Lucio Flores Payán*
}

Resumen: son diversos los enfoques y perspectivas sobre el desempeño de los gobiernos locales en México, y se han desarrollado formas diferentes para cuantificarlo, sin embargo, hay pocas investigaciones que presentan valoraciones de las tendencias nacionales sobre los cambios en el desempeño, y que se pueden percibir en un análisis comparativo en el tiempo. En el presente trabajo se propone la construcción de un índice según el enfoque de la teoría de la lógica difusa, que ha permitido obtener elementos para valorar el desempeño de los gobiernos locales. Se concluye que si bien éstos tienden a mejorar, no existe un patrón definido ni continuidad plena.

Palabras clave: gobiernos locales; desempeño gubernamental; lógica difusa.

Abstract: there are various approaches and perspectives on the performance of local governments in Mexico, and different ways to quantify this performance have been developed; however, there is little research showing an assessment of the national

\footnotetext{
Profesor-investigador en el Departamento de Políticas Públicas del Centro Universitario de Ciencias Económico Administrativas, de la Universidad de Guadalajara. Periférico norte Manuel Gómez Morín \#799, Núcleo Universitario Los Belenes, edificio B. C. P. 45100 Zapopan, Jalisco. Teléfono: (33) 3770 3300, extensión 25370. Correo electrónico: florespayan@ hotmail.com
} 
trends regarding changes in the performance of this type of governments, and that can be perceived in a comparative analysis over time. In this paper the construction of an index from the standpoint of the theory of fuzzy logic, which has enabled the production of elements to evaluate the performance of local governments is proposed. It follows that although local governments tend to improve their performance, there is no clear pattern, nor a full continuity.

Key words: local governments; government performance; fuzzy logic.

\section{Introducción}

Desde hace varias décadas, a los gobiernos municipales (GM) o también llamados locales (GL) de muchos países se les ha facultado para que tomen decisiones sobre una gran cantidad de deberes, con la finalidad de que sean responsables de responder a las sociedades mediante la administración de servicios públicos. Por tanto, su foco de atención es la administración de los recursos en torno a la eficiencia y el desempeño. En la literatura académica de las últimas décadas es evidente la afirmación del protagonismo de los GM en la promoción del desarrollo económico local. En este sentido, el estudio, análisis y evaluación del desempeño de los GL se torna cada vez más pertinente.

Este trabajo tiene dos objetivos principales, el primero es mostrar la evaluación de los gobiernos locales, que conforman las 32 entidades federativas de México, en el año 2000, 2005 y 2010, para medir su mayor o menor desempeño, a partir de la dimensión económica. El segundo es presentar una metodología alternativa de evaluación consistente en el diseño del índice difuso de desempeño de los gobiernos locales (IDDGL), basado en la teoría de la lógica difusa, mediante el cual se realiza el análisis multidimensional y multivariado a partir de una mayor exactitud en la parametrización y ponderación de los indicadores de medición.

Es pertinente mencionar que aquí no se pretende confrontar la metodología utilizada con ninguna otra diseñada para el cálculo de índices de desempeño, sino colaborar en la búsqueda de instrumen- 
tos que permitan la medición más precisa y adecuada del desempeño de los GL.Y, de esta forma, contribuir a la literatura sobre el tema, al presentar un análisis multidimensional, así como la postulación de una metodología alternativa para el diseño de índices de desempeño.

Este artículo contiene cuatro apartados, en el primero se exponen las características principales de los gobiernos locales y la medición de su desempeño, y se describe el estado del arte; en el segundo se explica puntualmente la metodología para el cálculo del IDDGL; en el tercero se muestran los resultados principales y al final se presenta una serie de conclusiones.

\section{El desempeño de los gobiernos locales}

A partir del análisis de los elementos teóricos del desempeño de los GL se pueden distinguir los determinantes presentados por Rainey y Steinbauer (1999), quienes proponen varios indicadores a partir de tres esferas principales: la disponibilidad de recursos, la cultura organizacional y el liderazgo. Por su parte, Boyne (2003) propone que el rendimiento de los GL puede explicarse a partir de la caracterización de los servicios públicos en términos de la cantidad de recursos, la reglamentación, las diferencias en la competencia del mercado, y los factores organizativos y de gestión.

Según estas dos líneas de análisis, Correa et al. (2013) cuestionan qué tan necesaria es la determinación del desempeño a partir del conglomerado de factores, o si es posible el análisis a partir de la evaluación de cada uno de estos elementos en su contribución individual al desempeño. En este sentido, la metodología propuesta aquí permitirá identificar la contribución de los indicadores analizados al valor total del desempeño de cada GL. Ferreira y Cunha (2013), en su revisión sistemática de la literatura sobre el tema, enfatizan en la pertinencia del esquema de eficiencia gubernamental de Renne (1937), entendiéndola como el valor de los servicios proporcionados por los gobiernos a partir de los ingresos obtenidos mediante la recaudación de impuestos, de igual manera una cantidad importante de trabajos que centraban la medición del desempeño a partir de la evaluación de un solo servicio o competencia, sin embargo, distinguen muchos 
otros donde se discute en la actualidad la necesidad y las dificultades de las mediciones del desempeño de los gobiernos locales a partir de metodologías más integrales y no unidimensionales.

En este sentido, en la práctica se han diseñado indicadores para la medición del desempeño gubernamental en las áreas siguientes:

- Fiscal. El propósito general de los indicadores fiscales es aproximar el esfuerzo de los gobiernos para acercar su recaudación al potencial de generación de ingresos y la posición fiscal.

- Financiera. Los indicadores financieros revelan la posición de las haciendas públicas en cuanto a sus fuentes de financiamiento, la asignación de recursos financieros, el balance entre éstos y el peso relativo que tienen sobre sus ingresos el gasto corriente, el costo financiero y el saldo de la deuda pública.

- Administrativa. Los indicadores administrativos muestran el desempeño en las funciones que se relacionan con la administración de los recursos humanos, financieros, físicos y tecnológicos del gobierno.

- Provisión de servicios. Estos indicadores evalúan en forma comparativa el desempeño de los gobiernos estatales o municipales en la producción y provisión de servicios, y se relacionan con los insumos empleados, los resultados obtenidos, la cobertura y el efecto en la sociedad.

- Económica. El objetivo de los indicadores económicos es mostrar la repercusión de la política económica del gobierno sobre los estados y municipios.

Para el caso mexicano, la literatura sobre municipios y su desempeño surge a partir de los procesos de descentralización que comenzaron en los primeros años de la década de 1980, sin embargo, fue en la siguiente cuando se concibieron los trabajos más representativos, entre los principales expositores están Cabrero (1996); Ziccardi (1995); Guillén (1996) y Sánchez (1998), quienes han aportado al tema elementos para la discusión primaria de la necesidad de gobernar a partir de los GL, así como la presentación de diseños metodológicos para medir y evaluar el desempeño gubernamental. 
Ejemplo de lo anterior es el trabajo de Sánchez y García (2001), donde se presenta un análisis del potencial de los GL para promover el desarrollo municipal, a partir de ejes de análisis como son las finanzas, la planeación, la participación ciudadana y la construcción de reglas para impulsar el desarrollo. Los autores concluyen que es muy reducida la capacidad institucional y organizacional de los GL para diseñar e instrumentar acciones efectivas en la promoción del desarrollo.

Por su parte, Sánchez et al. (2003) presentan el diseño y cálculo del índice de desempeño de los gobiernos locales (IDEGOB), el índice de delincuencia municipal y el índice de potencial del desarrollo municipal, para medir el desempeño de los municipios del estado de Jalisco. Para calcular cada índice se consideró una serie de indicadores, a partir de su ponderación y concentración sistemática. Para cada uno se presenta una clasificación que identifica una escala de cuatro niveles, de menor a mayor desempeño, para lo cual fueron clasificados los municipios analizados. Los autores afirman que ha sido poca la repercusión lograda por las políticas favorecedoras de la gestión y la administración locales.

Así mismo, se ha estudiado la participación ciudadana en la política pública municipal a partir de casos específicos, en esta línea están los trabajos de Ramírez Sainz (1998), quien argumenta que los GL la limitan y también la acción de las asociaciones civiles, con la intención de incrementar su posición de control lo que propicia una participación ciudadana simulada. Por su parte, Bazdresch (2003) plantea que en los procesos de decisión ésta se circunscribe a las actividades electorales en términos de votaciones. García (2006) ha estudiado cómo es que los esfuerzos gubernamentales por incorporar a los ciudadanos en la planeación del desarrollo y la gestión municipal se ven restringidos por la falta de capacidades de los GM, en donde éstas tienen que ver con los recursos humanos disponibles, la estructura y funciones organizacionales de los ayuntamientos y el marco institucional que regula la participación ciudadana en los municipios.

De acuerdo con la revisión de la literatura, se evidencia que el desempeño de los gobiernos locales puede determinarse por los resultados de las acciones dirigidas al mejoramiento de la sociedad que gobiernan, es así que trabajos como el de Ibarra et al. (2002) estable- 
cen las variables principales a partir de las cuales se puede medir el desempeño de los GL, proponen un índice integrado por indicadores que permiten evaluar servicios básicos como agua, drenaje, electricidad y de salud, educación y seguridad, igualmente contemplan indicadores de desempeño fiscal con base en la autonomía tributaria y capacidad recaudatoria, aspectos financieros como recursos para inversión o endeudamiento del municipio.

En este sentido, y a partir del argumento de que cualquier acción emprendida por los gobiernos locales se podrá evaluar como parte de los criterios del propio desempeño, el presente trabajo retoma las variables propuestas para el diseño de índices de desempeño como los de Ibarra et al. (2002) y en especial los de Sánchez et al. (2003), para la construcción y cálculo de un IDDGL.

Para tener un panorama más amplio de lo que ocurre con los gobiernos locales de México, se muestra lo obtenido de la estimación del IDDGL, esto permite tener resultados agregados que faciliten la comprensión de algunos elementos en la determinación del desempeño de los GL a partir de la llegada de actores, la instrumentación de nuevas visiones de gobierno y la aparición de políticas públicas locales durante el periodo 2000-2010.

\section{Metodología para el cálculo del IDDGL}

Antes de describir la metodología para calcular el IDDGL, se presentan algunos elementos de la teoría de la lógica difusa y su materialización en conjuntos difusos, sin la intención de profundizar en ellos, más bien se le da al lector interesado la opción de revisar los trabajos de Zadeh (1965); Flores y Camarena (2013) y Flores y García (2013), para conocer más sobre esta teoría y su aplicación práctica.

La teoría de la lógica difusa se materializa principalmente en metodologías para encontrar soluciones en procesos informáticos y de control en las áreas de ingeniería, debido a la posibilidad de trabajar con información imprecisa y con una vaguedad elevada, facultando la formalización y mesurado de información subjetiva a partir de la creación de un sistema de inferencia difuso (SID), que representa conocimientos y datos inexactos en forma similar a como lo hace el pensamiento humano. 
El SID se diseña a partir de la correspondencia no lineal entre una o varias variables de entrada y una de salida, pasa por una trasformación y recuperación de los datos (fuzzification-defuzzification), ${ }^{1}$ esto facilita una base desde la cual pueden tomarse decisiones o definir patrones (véase figura 1).

\section{Figura 1}

Etapas del sistema de inferencia difuso

\begin{tabular}{|c|c|c|}
\hline $\begin{array}{l}\text { Proceso de incorporación de } \\
\text { información } \\
\text { (fuzzification) }\end{array}$ & $\begin{array}{c}\text { Proceso de operaciones } \\
\text { recursivas } \\
\text { (reglas difusas) }\end{array}$ & $\begin{array}{l}\text { Proceso de respuesta o } \\
\text { salida de información } \\
\text { procesada al medio } \\
\text { (defuzzification) }\end{array}$ \\
\hline $\begin{array}{l}\text { A través de la lógica difusa, } \\
\text { los modelos de inferencia } \\
\text { difusa son capaces de } \\
\text { introducir a su dominio } \\
\text { de operación una gran } \\
\text { variedad de datos de diversa } \\
\text { naturaleza. Este proceso } \\
\text { implica una trasformación } \\
\text { de los datos perteneciente } \\
\text { a un dominio determinado } \\
\text { en términos de enunciados, } \\
\text { a los datos como valores } \\
\text { numéricos dentro } \\
\text { de variables en un } \\
\text { dominio difuso }\end{array}$ & $\begin{array}{l}\text { Dentro del sistema de } \\
\text { inferencia difusa, los } \\
\text { datos se encuentran ya } \\
\text { en un dominio numérico } \\
\text { y se trasmiten como } \\
\text { información para ser } \\
\text { procesada mediante } \\
\text { operaciones elementales } \\
\text { de diferenciación, } \\
\text { integración, comparación, } \\
\text { ponderación y repetición } \\
\text { formando funciones } \\
\text { representativas de } \\
\text { pertenencia a cada valor } \\
\text { lingüístico determinado }\end{array}$ & $\begin{array}{c}\text { En la salida del sistema } \\
\text { de inferencia difusa, } \\
\text { los valores resultantes } \\
\text { de las operaciones de } \\
\text { los conjuntos difusos } \\
\text { se trasladan de nuevo } \\
\text { a términos propios del } \\
\text { lenguaje del entorno }\end{array}$ \\
\hline
\end{tabular}

Fuente: Flores y Camarena (2013).

Zadeh presentó la representación matemática de esta teoría en 1965, a partir de la estructuración de conjuntos difusos, los cuales son una colección de objetos, donde a cada uno le corresponde una

1 Estos términos se toman del inglés, y se conservan en ese idioma porque aún no tienen traducción al español; su significado está expresado en la figura 1. 
función de membresía, que le asigna a un conjunto definido cierto grado de pertenencia. El rango de la función de pertenencia puede ser un conjunto de números reales no negativos, aunque la práctica general sea que la función de pertenencia esté definida entre 0 y 1 como $\mathrm{X} \mu_{\mathrm{A}} \rightarrow[1,0]$. Para ejemplificar esto, la representación matemática de un conjunto difuso, cuando $\mathrm{X}$ es una colección de objetos (universo) denotados por $\mathrm{x}, \mathrm{X}=\left\{\mathrm{x}_{1}, \mathrm{x}_{2}, \mathrm{x}_{3} \ldots\right\}$, así un subconjunto difuso $\mathrm{A}$ en $\mathrm{X}$ es un conjunto de pares ordenados:

$$
A=\{x, \mu A(x), \mid x \in U\}
$$

donde $\mu_{\mathrm{A}}$ : $[\theta$ es la función de pertenencia, $\mu \mathrm{A}(x)$ es el grado de pertenencia de la variables x y $\mathrm{U}$ es el dominio de la aplicación, en términos difusos es lo referente al universo de estudio, es decir, mientras más cercano sea el valor de " $\mu \mathrm{A}(x)$ " a la unidad, mayor será la pertenencia del objeto $\mathrm{x}$ al conjunto A $\mathrm{o}$.

Cuando el universo de estudio esté configurado de forma continua o discreta serán representados respectivamente por las siguientes ecuaciones:

$$
\mathrm{A}=\frac{\int_{\mathrm{x}}(\mu \mathrm{A}(\mathrm{x})}{\mathrm{x}} \quad \mathrm{A}=\sum_{i=1}^{n} \frac{\mu \mathrm{A}(\mathrm{xi})}{\mathrm{xi}}
$$

Sistema de inferencia difuso para el cálculo del IDDGL

El cálculo del IDDGL se realiza mediante la construcción de conjuntos difusos, para cuantificar y valorar la actuación de los GM, que aquí se denomina "desempeño de los gobiernos locales". Para lograr dicho objetivo se propone la construcción de un IDDGL, para lo que se toma como referencia el índice propuesto por Sánchez et al. (2003). El IDDGL es alimentado por cuatro ejes de análisis, construidos a partir de las siguientes funciones y sus variables respectivas:

Eje 1: oferta de servicios (os)

OS=f (cobertura de agua, cobertura de drenaje, cobertura de electricidad)

Eje 2: situación financiera (SF)

$S F=f$ (porcentaje de ingresos propios, presión fiscal) 
Eje 3: eficiencia de gasto (EG)

$E G=f$ (porcentaje de gasto administrativo, porcentaje de ingresos propios con respecto al gasto administrativo

Eje 4: creación de infraestructura (CI) $C I=f$ (porcentaje de gasto en obra pública)

Para cada eje se construyó un SID, que se compone de conjuntos difusos para representar a cada variable de los ejes de análisis, que usan etiquetas lingüísticas para reflejar el grado de desempeño en un continuo. Para explicar la construcción de los conjuntos difusos asociados al eje 1, se utilizó el software Matlab.

Cada variable de entrada y de salida se compone de tres conjuntos difusos, que representan las etiquetas lingüísticas del rango total de la variable de entrada (véase figura 2). Cada conjunto difuso construido

\section{Figura 2}

Conjuntos difusos para cada variable del SID del eje 1
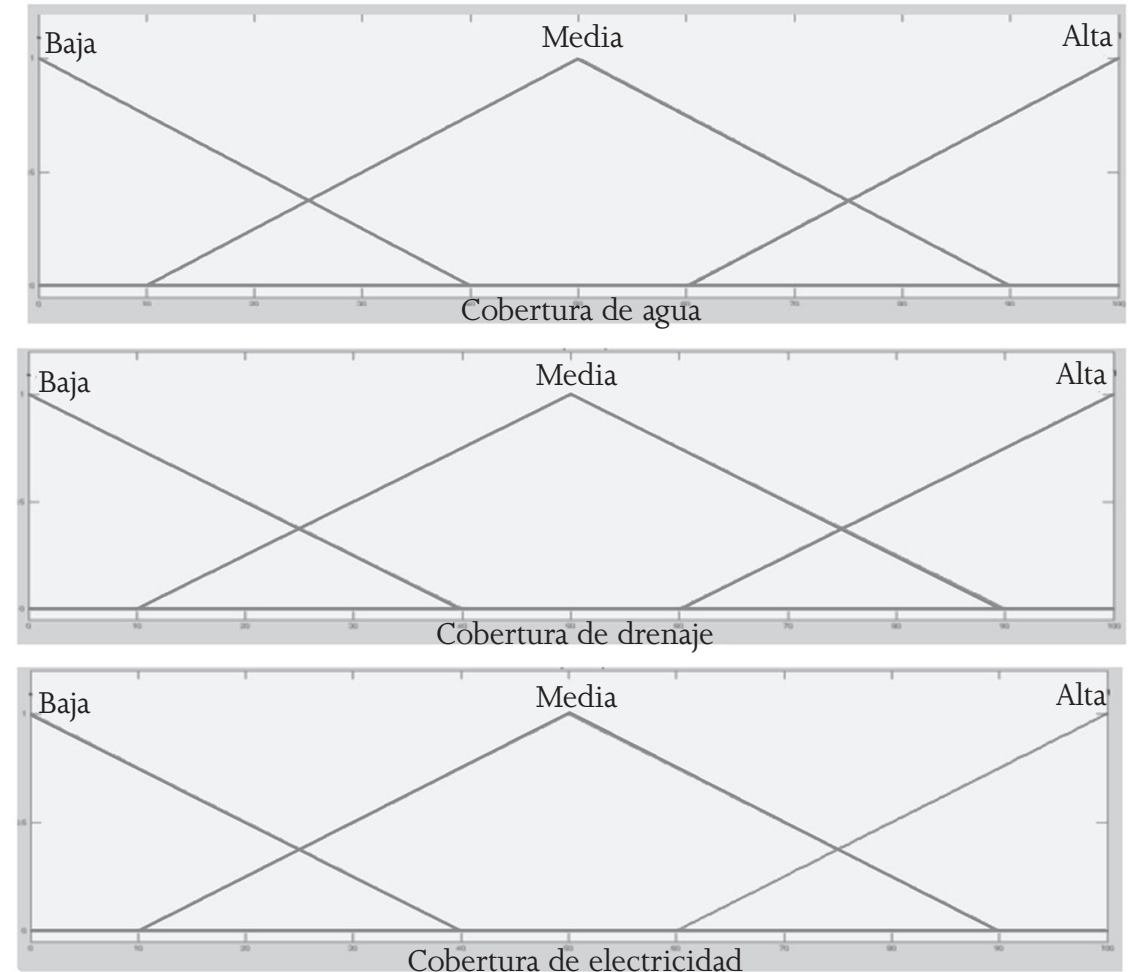

Fuente: elaboración propia. 


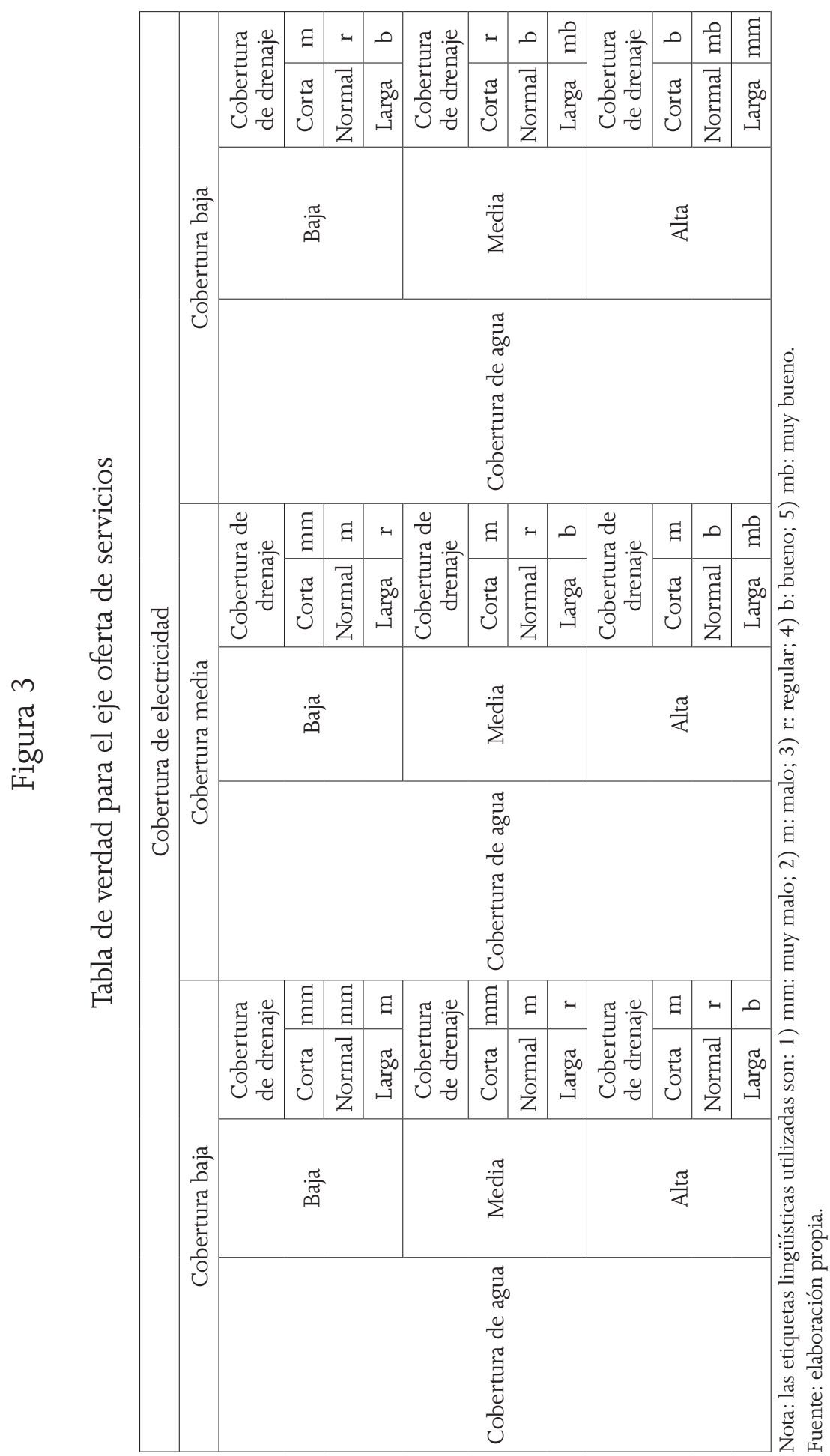


para cada variable del siD se conforma por tres funciones de pertenencia, marcadas con tres etiquetas lingüísticas; para la de "cobertura de agua” se establecen las siguientes: "baja”, "media” y "alta”.

En el proceso de fuzzification-defuzzification se requiere la construcción de las reglas de operación del SID, que permitan establecer los criterios de valoración sobre los cuales se hará el cálculo a través de parámetros de referencia basados en las etiquetas lingüísticas. Para esto se construyó una tabla de verdad para cada eje de análisis (véase figura 3).

Con estos dos insumos: conjuntos difusos y sus reglas de operación respectivas (tabla de verdad), se procedió a la etapa de fuzzification-defuzzification, se empleó el método Mamdani y la herramienta de fuzzy logic en el software matemático Matlab. En la figura 4 se muestra el modelo de fuzzification para el eje de oferta de servicios.

Figura 4

Sistema de inferencia difuso del eje oferta de servicios

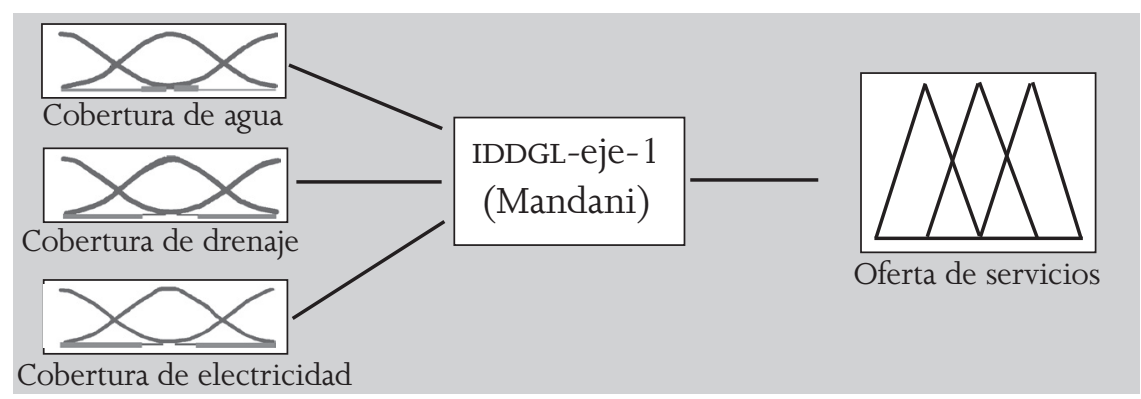

Fuente: elaboración propia.

El proceso de defuzzification se conformó por una variable de salida del sID para la cual se contemplaron cinco funciones de pertenencia, clasificadas con las etiquetas lingüísticas de: "muy mala", "mala", "regular", "buena" y "muy buena", como se ilustra en la figura 5, para obtener una ubicación clara del valor final del IDDGL. 


\section{Figura 5}

Conjuntos difusos para la variable de salida del eje oferta de servicios

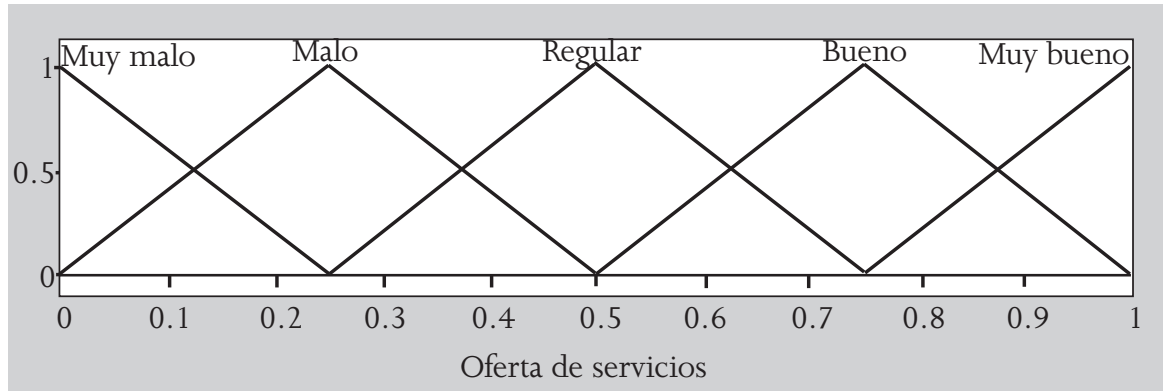

Fuente: elaboración propia.

Para alimentar las funciones de los SID de cada eje de análisis y el cálculo del IDDGL, se tomaron datos del Sistema Estatal y Municipal de Bases de Datos (SIMBAD s/f) del Instituto Nacional de Estadística y Geografía, para el año 2000, 2005 y 2010. La selección de la muestra de GL fue a partir del criterio de correspondencia con la información requerida para la alimentación del sistema de inferencia difusa, que a su vez estuviera disponible para todo el periodo de análisis, debido a esto, de todos los municipios de México la muestra se acotó a 1523 , si bien lo anterior fue una limitante en términos de la representatividad estadística que pudiera alcanzarse, para el presente trabajo no implicó gran problema debido a que el interés principal fue mostrar las tendencias en términos del desempeño de los GL mexicanos.

El desempeño de los gobiernos municipales 2000,2005 y 2010

El procedimiento para calcular el IDDGL arrojó resultados consistentes, en relación con los trabajos empíricos presentados en el primer apartado. Sin embargo, los valores obtenidos a partir de las propuestas de un índice difuso propiciaron información adicional a partir de la facilidad para calcular el índice de forma más desagregada y multi- 
dimensional del IDDGL para los 1523 gobiernos locales, para 2010, 2005 y $2000 .^{2}$

Para mostrar de forma más clara el comportamiento en términos del desempeño de los GL, se estableció una categorización con las cinco etiquetas lingüísticas que agrupan el rango total del IDDGL. Éstas se calcularon a partir de un procedimiento de estadística simple, que corresponde al criterio mostrado en la figura 6, donde cada uno se encuentra en función de la media y la desviación estándar de la integración del total del IDDGL de los GL analizados.

\section{Figura 6}

Etiquetas de desempeño para los gobiernos locales calculados con el IDDGL

\begin{tabular}{|c|c|}
\hline Desempeño & Criterio \\
\hline Muy bueno & $\mathrm{X}>(\overline{\mathrm{X}}+26)$ \\
\hline Bueno & $(\overline{\mathrm{X}}+6)<\mathrm{X}<(\overline{\mathrm{X}}+26)$ \\
\hline Regular & $(\overline{\mathrm{X}}-6)<\mathrm{X}<(\overline{\mathrm{X}}+6)$ \\
\hline Malo & $(\overline{\mathrm{X}}-26)<\mathrm{X}<(\overline{\mathrm{X}}-6)$ \\
\hline Muy malo & $\mathrm{X}<(\overline{\mathrm{X}}-26)$ \\
\hline Nota: $\bar{X}:$ media aritmética, 6: desviación estándar & \\
\hline
\end{tabular}

Fuente: elaboración propia.

La estimación del IDDGL reporta que el mayor porcentaje de gobiernos locales cae en la categoría de desempeño regular para los tres periodos, sin embargo, puede apreciarse que en la de malo se ha incrementado significativamente, con una tendencia a la estabilidad en el regular, para 2000-2005, en tránsito hacia el malo y regular, en 2010 (véase figura 7).

Con la idea de visualizar el empeoramiento o mejoramiento del desempeño de los GL, en la figura 8 se presenta un comparativo porcentual para cada categoría, se aprecia que para el total del periodo

2 Por cuestiones de espacio, en el presente artículo no fue posible incluir el listado de los valores obtenidos a partir del IDDGL. Si el lector tiene interés en consultarlos, los puede solicitar al autor. 


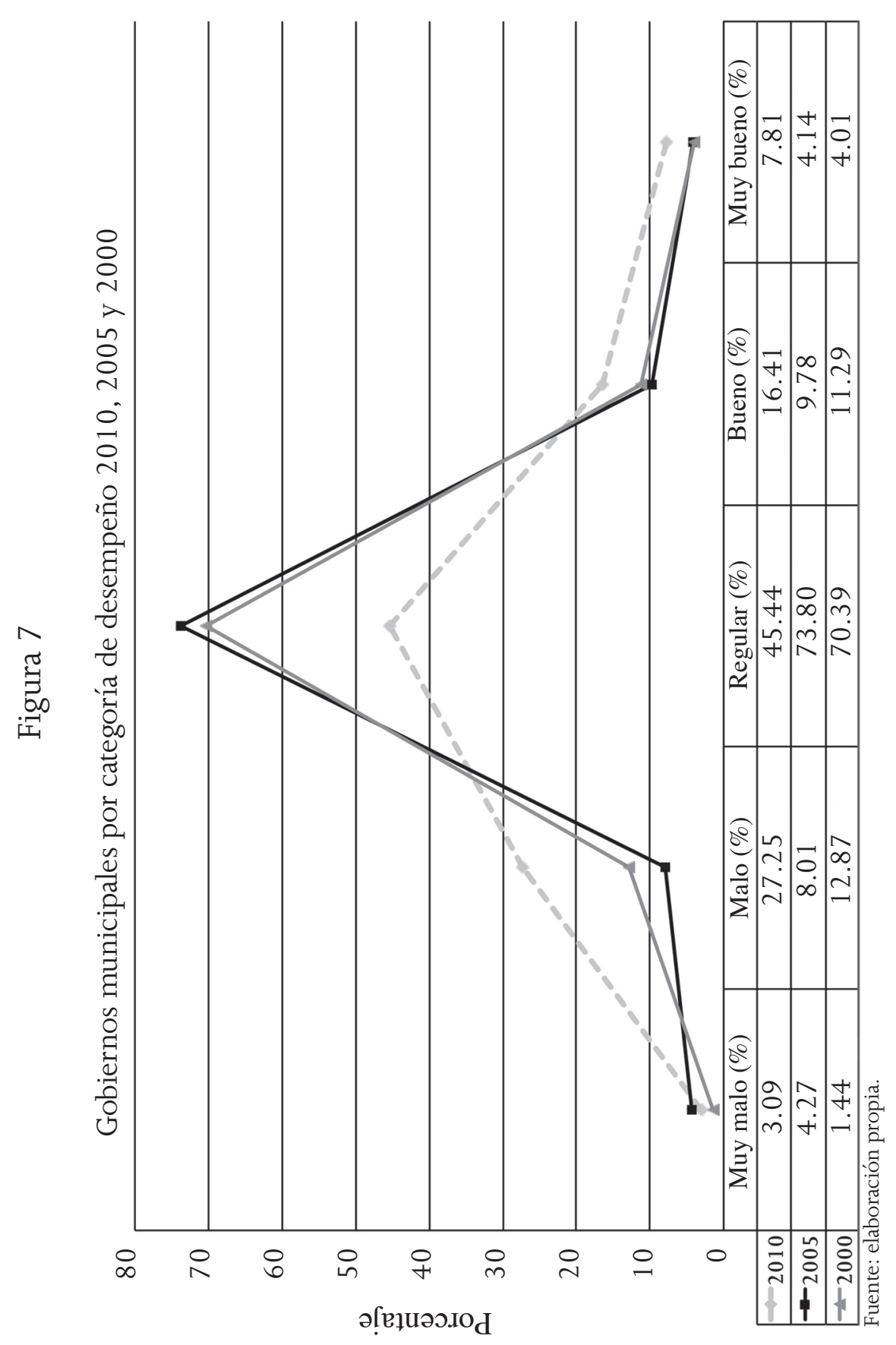




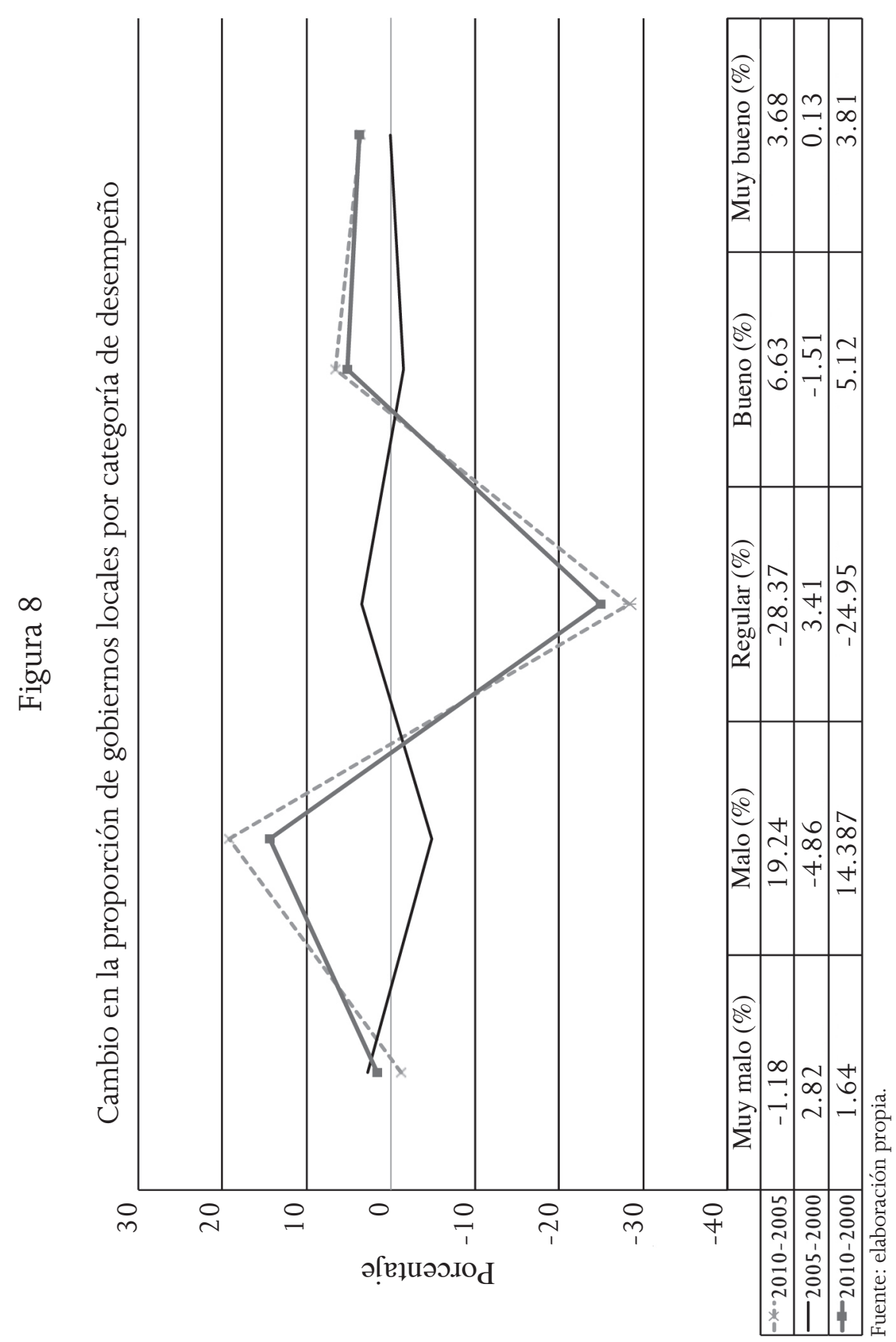


2010-2000, la de regular tendió a reducirse, esto se podría interpretar como que el desempeño de los GL en general ha tratado de estabilizarse, sin embargo, no alcanza mejoras, se puede observar que para 2010-2005, los GL con desempeño malo se incrementaron en 19.24 por ciento, y para 2010-2000 aumentaron 14.38 por ciento en esta categoría. Así mismo, se aprecia que para las de "bueno" y "muy bueno" no se detectan grandes incrementos ni siquiera en el periodo total, lo que podría indicar, tanto en el corto como en el largo plazo, que los GL tienen problemas para estabilizar su desempeño, los cambios porcentuales mostrados para cada categoría suponen que dicho desempeño es totalmente inestable, y que transitan de uno a otro sin seguir una línea de continuidad.

A partir del supuesto de que los gobiernos con capacidades de gestión más desarrolladas establecen políticas públicas con mayor efectividad, que les permite lograr más desarrollo $y$, por consiguiente, un mejor desempeño gubernamental, Guillén (1996) hace puntualizaciones evidentes de que entre más grandes sean los municipios (urbanos y metropolitanos), alcanzarán más capacidades y posibilidades para la innovación en la gestión, lo que se ve reflejado en mejores desempeños. En este sentido, se calculó el IDDGL a partir del tamaño de los municipios (véase figura 9).

\section{Figura 9}

Porcentaje de municipios por su tamaño, que mejoraron su IDDGL

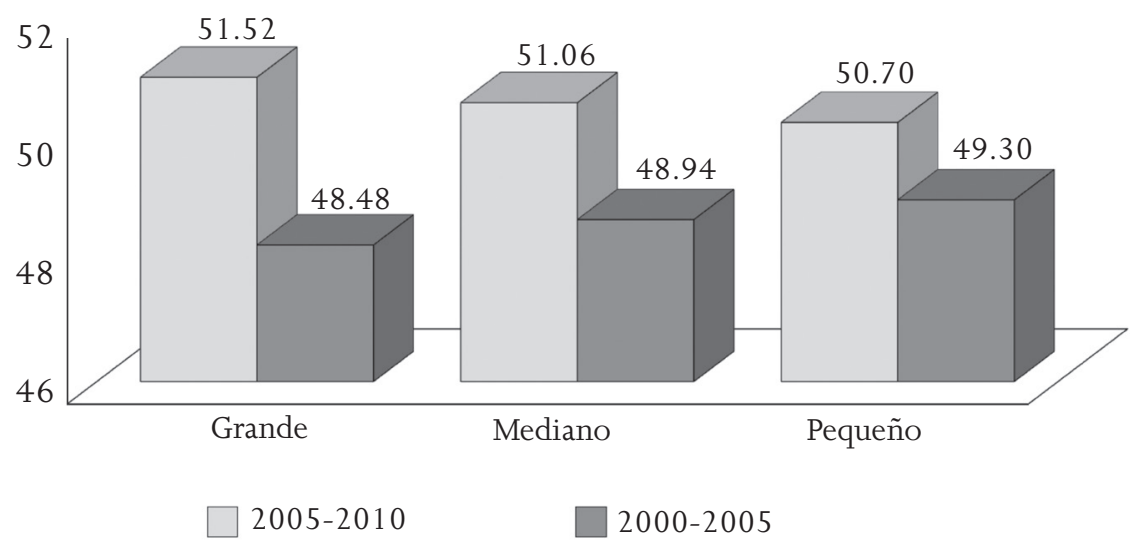

Fuente: elaboración propia. 
En ninguno de los periodos de análisis los municipios más grandes incrementaron su desempeño. Para 2000-2005, el cambio en el IDDGL fue muy similar para los tres tipos de municipios, aproximadamente 50 por ciento, mientras que para 2005-20010 fue de 49.30, que corresponde a los pequeños que lograron mejorar en mayor proporción. Lo anterior es evidencia de que el supuesto que atribuye más desempeño a los municipios con mayor tamaño no se corrobora a plenitud.

En la figura 10 aparece el esquema de la frecuencia de las categorías del IDDGL, donde resalta que hubo más GL en la categoría de regular, y muy poca frecuencia en la de muy malo.

Con la intención de presentar de forma más puntual y en mayor medida multidimensional, se calculó el IDDGL por tamaño del muni-

Figura 10

Frecuencia de las categorías del IDDGL 2010

Categoría del IDDGL 2010
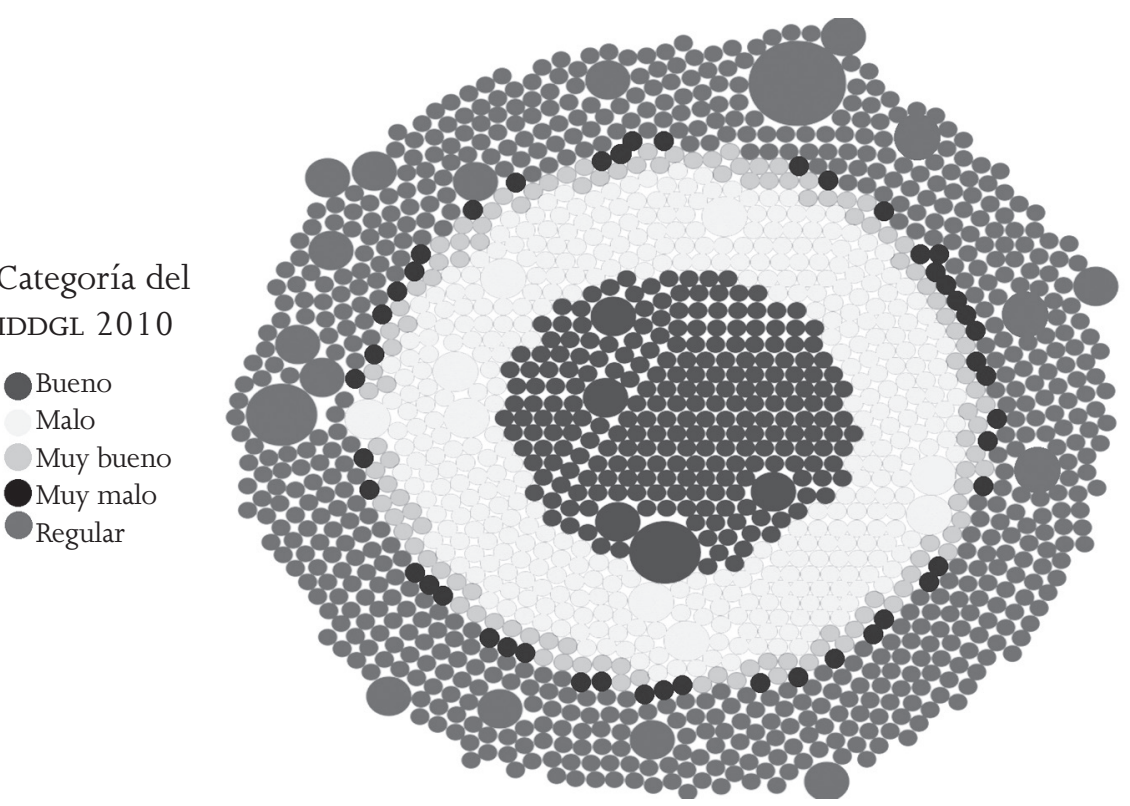

Fuente: elaboración propia. 
cipio para cada categoría de desempeño (véase figura 11); se aprecia que el porcentaje más alto es en la de regular, en mayor medida para todo el periodo. Para 2010 éste cayó en la de regular, con 47.06 por ciento, y corresponde a los municipios medianos, mientras que para 2005 y 2000, el mayor porcentaje también fue de regular para los grandes y medianos respectivamente.

\section{Figura 11}

Desempeño de los gobiernos por tamaño de municipio

$$
2000,2005 \text { y } 2010
$$

\begin{tabular}{|l|r|r|r|r|r|r|r|r|r|}
\hline \multirow{2}{*}{ Categoría } & \multicolumn{3}{|c|}{$2010(\%)$} & \multicolumn{3}{c|}{$2005(\%)$} & \multicolumn{3}{c|}{$2000(\%)$} \\
\cline { 2 - 10 } & Grande & Mediano & Pequeño & Grande & Mediano & Pequeño & Grande & Mediano & Pequeño \\
\hline Muy malo & 3.62 & 3.27 & 2.48 & 1.29 & 1.80 & 1.15 & 0.52 & 1.80 & 1.72 \\
\hline Malo & 24.81 & 26.96 & 29.39 & 7.75 & 9.97 & 14.12 & 12.14 & 10.46 & 16.22 \\
\hline Regular & 45.22 & 47.06 & 43.70 & 75.19 & 73.37 & 71.95 & 68.22 & 73.20 & 68.70 \\
\hline Bueno & 17.31 & 15.52 & 16.79 & 12.14 & 10.95 & 8.78 & 13.18 & 11.76 & 9.35 \\
\hline Muy bueno & 9.04 & 7.19 & 7.63 & 3.62 & 3.92 & 4.01 & 5.94 & 2.78 & 4.01 \\
\hline
\end{tabular}

Fuente: elaboración propia.

Pareciera que el desempeño de los gobiernos locales siempre está situado en la categoría de regular, sin embargo, al hacer una observación segmentada del periodo total de análisis en dicha categoría se visualiza una tendencia a la baja, con una cambio porcentual negativo para 2005-2010 de -30 por ciento para los tres tamaños de municipio. También es relevante el cambio porcentual en la etiqueta de desempeño malo, ya que 17.05 por ciento de los municipios grandes se situaron en esta categoría. Con este análisis se puede aseverar que si bien los GL han logrado mejoras en su desempeño, no se podría establecer criterios para argumentar que el tamaño influye fuertemente en él.

Los valores del IDDGL promedio también se estimaron con la idea de mostrar el desempeño de los GL para cada eje de análisis, lo cual 

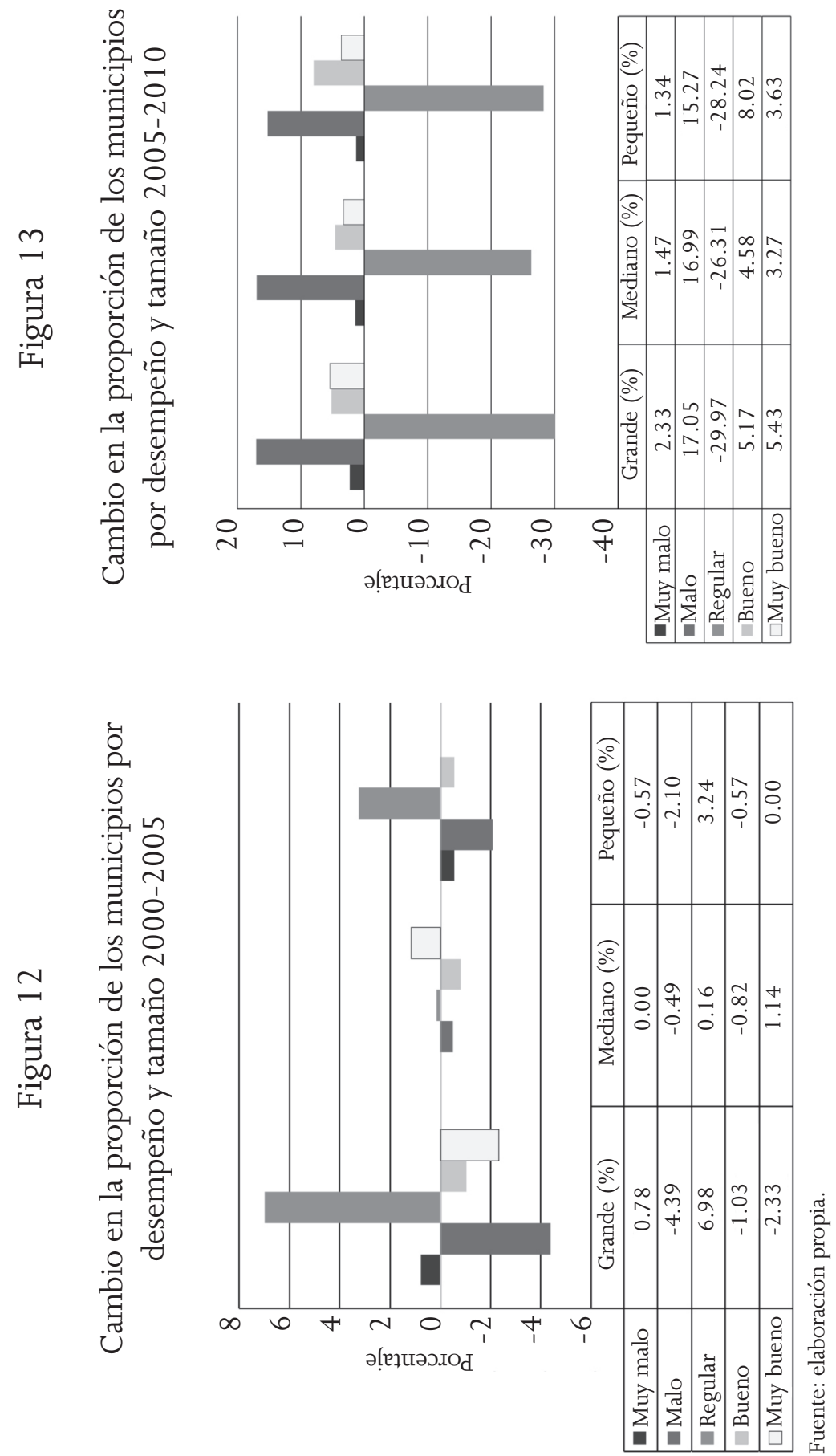
es factible debido al uso del modelo de lógica difusa, en la figura 14 se aprecia que el más alto corresponde a las actividades en el eje 1, el de la oferta y servicios, con un valor de 0.6149 , esto es evidencia de que para todo el periodo este eje alcanzó los valores más elevados del IDDGL.

\section{Figura 14}

IDDGL promedio por eje y tamaños de los municipios para el periodo de análisis

\begin{tabular}{|c|c|r|r|r|r|}
\hline Año & Tamaño del municipio & \multicolumn{1}{|c|}{ Eje 1 } & \multicolumn{1}{c|}{ Eje 2 } & \multicolumn{1}{c|}{ Eje 3 } & \multicolumn{1}{c|}{ Eje 4 } \\
\hline \multirow{3}{*}{2010} & Grande & 0.6149 & 0.1286 & 0.4674 & 0.2799 \\
\cline { 2 - 6 } & Mediano & 0.5743 & 0.04704 & 0.3602 & 0.3375 \\
\cline { 2 - 6 } & Pequeño & 0.5661 & 0.0319 & 0.3562 & 0.268 \\
\hline \multirow{3}{*}{2005} & Grande & 0.5088 & 0.1336 & 0.4832 & 0.2799 \\
\cline { 2 - 6 } & Mediano & 0.5221 & 0.0512 & 0.3822 & 0.3375 \\
\cline { 2 - 6 } & Pequeño & 0.5282 & 0.0343 & 0.3784 & 0.268 \\
\hline \multirow{3}{*}{2000} & Grande & 0.4984 & 0.0753 & 0.4177 & 0.2498 \\
\cline { 2 - 6 } & Mediano & 0.4953 & 0.0653 & 0.246 & 0.3055 \\
\cline { 2 - 6 } & Pequeño & 0.4908 & 0.082 & 0.4137 & 0.1975 \\
\hline
\end{tabular}

Fuente: elaboración propia.

En las figuras 15 y 16 se muestran las regiones que tuvieron los valores más altos de desempeño para los GL, es evidente un cambio notorio para el año 2000, cuando la mayoría de municipios se concentraron con uno regular en el noreste, occidente y centro norte, en tanto en el norte y sur sostuvieron uno malo, para 2010 los GL del occidente y centro norte tuvieron un despunte, se aprecia un mejoramiento en el IDDGL también para los de la región norte, en tanto la sur siguió con valores más bajos del IDDGL.

Con la intención de mostrar la complementariedad entre la metodología utilizada en este trabajo y otras, para la construcción de índices de desempeño gubernamental, se hizo un esfuerzo por mostrar una comparación en términos analíticos de los resultados obtenidos por Sánchez y García (2001), cuyo trabajo se usó para realizar 


\section{Figura 15}

Ubicación de los municipios de acuerdo con el valor de su IDDGL en el 2010

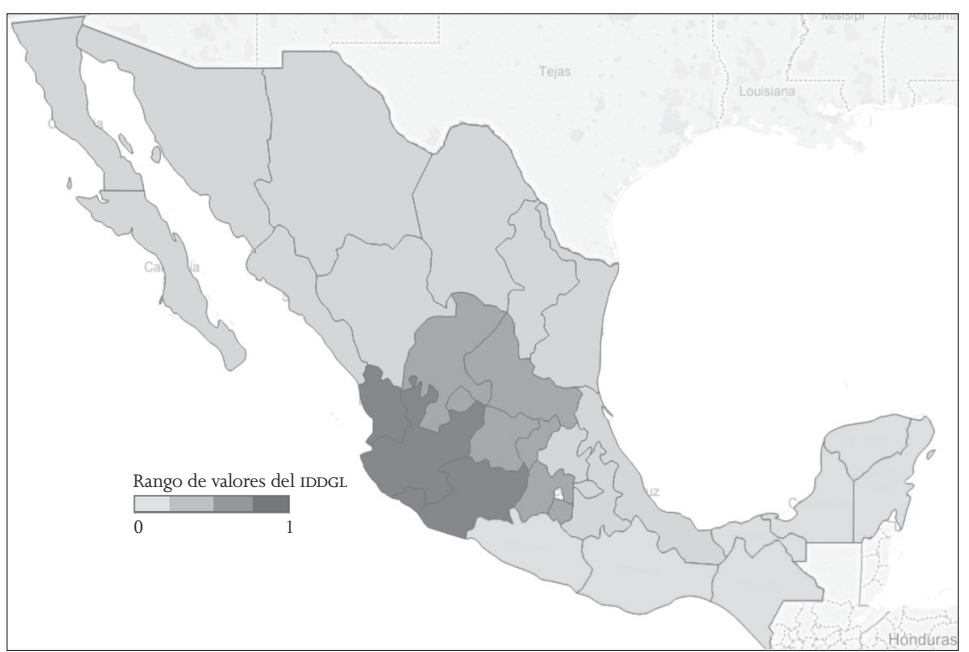

Fuente: elaboración propia.

Figura 16

Ubicación de los municipios de acuerdo con el valor de su IDDGL en el año 2000

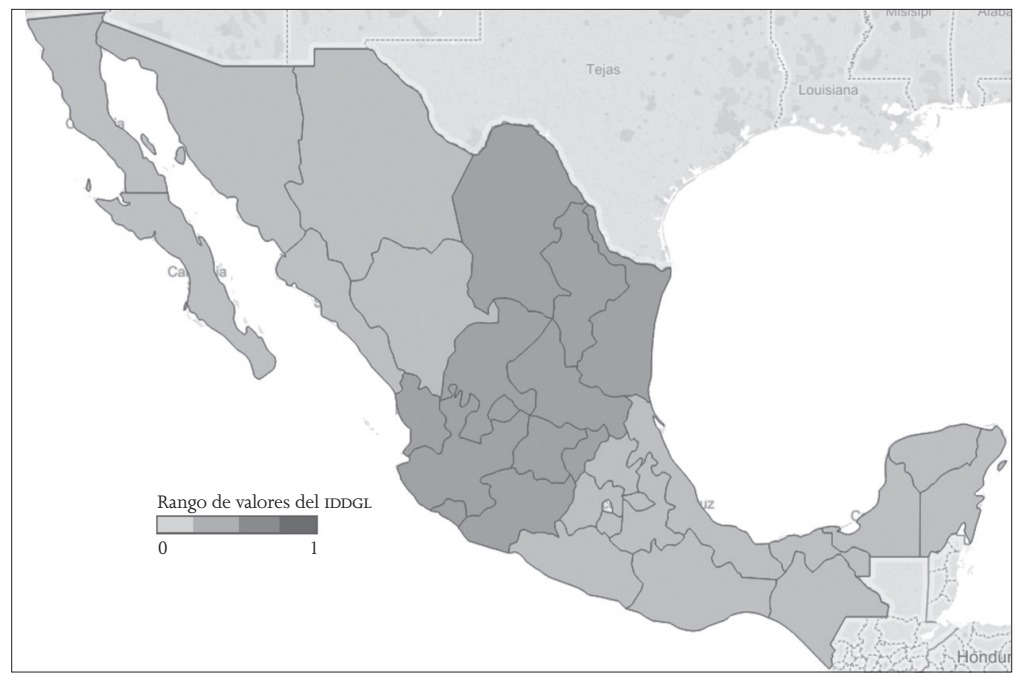

Fuente: elaboración propia. 
el comparativo, debido a su proximidad en los periodos de análisis y la similitud en las variables empleadas, sin embargo, es pertinente mencionar que para lograr una comparación más exacta se requiere mayor precisión en el periodo de medición del desempeño (véase figura 17).

\section{Figura 17}

\section{Comparativo IDEGOB versus IDDGL}

\begin{tabular}{|c|c|c|}
\hline Índice & IDEGOB & IDDGL \\
\hline $\begin{array}{l}\text { Forma de } \\
\text { cálculo }\end{array}$ & $\begin{array}{c}\text { • Suma de los valores de } \\
\text { los indicadores, a su vez cada } \\
\text { indicador obtiene un valor } \\
\text { como resultado de la suma } \\
\text { del puntaje de las variables } \\
\text { que los componen, y estas } \\
\text { puntuaciones son asignadas. Los } \\
\text { valores o puntajes que otorgan } \\
\text { los indicadores y la variables al } \\
\text { cálculo del índice de desempeño } \\
\text { del GM en Jalisco se estimaron } \\
\text { con el método Delphi }\end{array}$ & $\begin{array}{l}\text { - Sistema de inferencia difusa } \\
\text { a partir de la construcción } \\
\text { de conjuntos difusos, } \\
\text { para la parametrización } \\
\text { de las variables } \\
\text { de cada indicador }\end{array}$ \\
\hline Resultados & $\begin{array}{l}\text { • Muestran un incremento del } \\
\text { porcentaje de GL en la categoría } \\
\text { de "desempeño malo" } \\
\text { • Los GL en municipios } \\
\text { clasificados como grandes } \\
\text { alcanzan valores más elevados } \\
\text { en el índice de desempeño }\end{array}$ & $\begin{array}{l}\text { • Muestran una reducción } \\
\text { del porcentaje de GL en la } \\
\text { categoría de "desempeño malo" } \\
\text { • Los GL en los municipios } \\
\text { clasificados como grandes } \\
\text { y pequeños alcanzan valores } \\
\text { similares del índice de desempeño }\end{array}$ \\
\hline Conclusiones & $\begin{array}{l}\text { - No presenta una tendencia } \\
\text { clara y contundente que permita } \\
\text { afirmar que la descentralización } \\
\text { ha generado un entorno } \\
\text { adecuado para mejorar } \\
\text { el desempeño de los GM; } \\
\text { por el contrario, se encontraron } \\
\text { comportamientos erráticos } \\
\text { y poco alentadores. La mayoría } \\
\text { de éstos no evidenciaron } \\
\text { mejoras en el desempeño; } \\
\text { más de } 50 \% \text { de ellos se ubican } \\
\text { en las categorías de desempeño } \\
\text { regular y malo }\end{array}$ & $\begin{array}{l}\text { - Comportamiento irregular en } \\
\text { el desempeño de lo GL, es decir, } \\
\text { no hay una tendencia clara al } \\
\text { mejoramiento pero tampoco su } \\
\text { decremento, no existe un patrón }\end{array}$ \\
\hline
\end{tabular}

Fuente: elaboración propia. 
Son evidentes las similitudes y también las divergencias en los resultados, sin embargo, se puede destacar que en cuanto al uso de la lógica difusa en la construcción de índices de desempeño, los resultados son consistentes y robustos, igual que para el caso del IDEGOB, con la ventaja de no tener que recurrir a la valoración de expertos para la ponderación de los parámetros e indicadores que se contemplan en el índice, de tal manera que simplifica el proceso de cálculo y con fuentes de información, como bases de datos, podría generarse un sistema de inferencia difusa que calculara de forma automática esta ponderación. Así mismo, usar un índice construido con base en la lógica difusa permitirá un análisis multidimensional, sin que se alteren los valores del índice.

\section{Conclusiones}

Con base en el análisis realizado y la información presentada, es factible afirmar que el estudio del desempeño de los gobiernos locales requiere de indicadores más precisos, que den cuenta de elementos fundamentales, mediante los cuales éstos establezcan criterios de focalización para mejorar su desempeño. La generación de índices, como el estimado en el presente trabajo, permite conocer la evolución que los GM logran en términos de su desempeño, y así tener referentes sobre el que se produce en las regiones de México, a partir de sus GL.

Los resultados obtenidos permitieron identificar el comportamiento de los GL en relación con su desempeño, es evidente que es sumamente irregular, es decir, no existe una tendencia clara al mejoramiento pero tampoco al decremento. El patrón de comportamiento es muy variante, para el primer corte, correspondiente a 2000-2005, en el que los municipios grandes tuvieron mayor proporción de desempeño regular, sin embargo, también en un gran porcentaje de éstos el desempeño fue muy malo. Con respecto al segundo corte, 20052010, en los GL hubo avances en la categoría de muy bueno para los tres tamaños de municipio, sin embargo se pasó de uno regular, en el primer periodo, a uno malo, puesto que un gran porcentaje de GL se situó en esta categoría. 
Es pertinente mencionar la factibilidad de la implementación, el diseño y la estimación de los índices de desempeño, a partir de los elementos de la lógica difusa, en comparación con los índices convencionales, el IDDGL permite hacer estimaciones de forma multidimensional y puntualizar una para cada eje analizado. A manera de conclusión, se puede precisar que el IDDGL es un aporte que complementa al resto de los índices y estrategias metodológicas diseñadas para la medición y cuantificación del desempeño de los gobiernos locales.

Recibido en noviembre de 2014 Aceptado en marzo de 2015

\section{Bibliografía}

Bazdresch, Miguel. 2003. Cambio municipal y participación social. En Políticas públicas municipales. Una agenda en construcción, compilado por Enrique Cabrero Mendoza, 49-86. México: Miguel Ángel Porrúa y Centro de Investigación de Docencia Económicas (CIDE).

Boyne, George. 2003. Sources of public service improvement: a critical review and research agenda. Journal of Public Administration Research and Theory: 367-394.

Cabrero Mendoza, Enrique. 1996. Las políticas descentralizadoras en el ámbito internacional. Análisis de tendencias y obstáculos en diversos países. Nueva Sociedad (142): 72-95.

Cabrero Mendoza, Enrique. 1995. La nueva gestión municipal en México. Análisis de experiencias innovadoras en gobiernos locales. México: Miguel Ángel Porrúa y CIDE.

Correa Gomes, Ricardo, Solange Alfinito y Pedro Melo Alburquerque. 2013. Analyzing local government financial performance: evidence form Brazilian municipalities 2005-2008. ANPAD 17(6): 704-719. 
Ferreira da Cruz, Nuno y Rui Cunha Marques. 2013. Revisiting the determinants of local government performance. ELSEVIER: 91-103.

Flores Payán, Lucio y Margarita Camarena Luhrs. 2013. Evaluación de programas públicos en el marco de la realidad social. Metodología basada en la lógica difusa como instrumento para el análisis de fenómenos sociales. Revista Latinoamericana de Metodología de la Investigación Social-Relmis 5: 8-23.

Flores Payán, Lucio y María Luisa García Bátiz. 2013. Evaluación de programas públicos mediante lógica difusa. El caso del programa Hábitat. Revista Política y Cultura 40: 231-255.

García Bátiz, María Luisa. 2006. Planeación participativa. La experiencia de la política ambiental en México. México: Plaza y Valdez.

García Bátiz, María Luisa, Sergio M. González Rodríguez, Antonio Sánchez Bernal y Basilio Verduzco Chávez. 1998. Descentralización e iniciativas locales de desarrollo. Guadalajara: Universidad de Guadalajara, Universidad de California en Los Ángeles y Juan Pablos Editor.

Guillén López, Tonatiuh. 1996. Gobiernos municipales en México: entre la modernización y la tradición política. México: Miguel Ángel Porrúa y El Colegio de la Frontera.

Ibarra Salazar, Jorge, Alfredo Sandoval Musi, Lidia Sotres Cervantes y Rodrigo Pérez Alanís. 2002. Desempeño del gobierno en los municipios mexicanos. Monterrey: División de Administración y Finanzas, Departamento de Economía, Instituto Tecnológico y de Estudios Superiores de Monterrey.

Rainey, Hal y Paula Steinbauer. 1999. Galloping elephants: developing elements of a theory of effective government organizations. Journal of Public Administration Research and Theory 9 (1): 1-32.

Ramírez Sainz, Juan Manuel. 1998. Cómo gobiernan Guadalajara. Guadalajara: Miguel Ángel Porrúa, Universidad Nacional Autónoma de México-Instituto de Investigaciones Sociales y Universidad de Guadalajara. 
Renne, R. 1937. Researchin measring the efficiency of local governments. Journal of Farm Economics 19: 553-557.

Sánchez Bernal, Antonio. 1998. Relevancia de los estudios en desarrollo municipal. El caso de los municipios mexicanos. Revista Interamericana de Planificación Xxx (117-118): 238-250.

Sánchez Bernal, Antonio y María Luisa García. 2001. Libertad y desarrollo económico local. En La ruta del cambio institucional, compilado por Antonio Sánchez Bernal, 53-76. México: Universidad de Guadalajara.

Sánchez Bernal, Antonio, Édgar Tovar García y Antonia Sánchez Martínez. 2003. Evaluación del desempeño de los gobiernos municipales de Jalisco. Hacia una política regional de fortalecimiento municipal. Gestión Municipal 1 (1): 55-72.

SIMBAD. (s/f). INEGI. http://www.inegi.org.mx/prod_serv/contenidos/espanol/simbad/default.asp?c $=73$

Ziccardi, Alicia. 1995. La tarea de gobernar: gobiernos locales y demandas ciudadanas. México: Miguel Ángel Porrúa.

Zadeh, Lofti. 1965. Fuzzy sets. Department of Electrical Engineering and Electronics Research Laboratory. Universidad de California en Berkeley. 